\title{
A risk evaluation model for channel navigation based on the gray-fuzzy theory
}

Yanfeng Wang ${ }^{1,2,3^{*}}$, Liwen Huang ${ }^{1,2}$, Guohua Shen ${ }^{3}$ and Mingming Jia ${ }^{1,4}$

\begin{abstract}
In view of the fuzziness and randomness of the safety evaluation of the channel navigation environment, a risk evaluation model of the channel navigation is established with the set-valued statistics and the gray theory. A safety evaluation index system of channel navigation consisting of first-level indexes for the natural environment, navigation conditions, and traffic environment as well as $12 \mathrm{~s}$-level indexes were established on the basis of comprehensively analyzing factors influencing the safety of the channel navigation environment. The weight of each evaluation index is determined through the model utilizing the set-valued statistics; also, the evaluation sample matrix is determined to realize the initial construction of the environmental scenario of channel navigation; and the safety of the channel navigation environment is evaluated quantitatively with the gray fuzzy comprehensive evaluation method. Taking Guangdong inland channel as an example, the result shows that the safety evaluation method based on the set-valued statistics and the gray fuzzy theory features simple calculation as well as objective and accurate results, which is convenient for practical application.
\end{abstract}

Keywords: Channel, Set-valued statistics, The gray-fuzzy theory, Risk evaluation, System model

\section{Introduction}

The channel is an important part of the water transport system, whose navigation environment has a significant impact on the safe navigation of the ship. Frequent accidents of channel navigation have not only caused great losses to the country, but also hindered the sustainable development of channels. How to protect the safety of shipping navigation environment is an important research work on safety management of shipping channel. There are many factors affecting the safety of the channel navigation environment. In order to ensure the safety of channel navigation, these influencing factors should be analyzed and studied to provide a scientific and reasonable judging basis for the safety evaluation of the channel navigation environment.

A variety of research have been made on the channel navigation environment by many professionals. Wang et al. [1] not only proposed a DEA model algorithm, but also analyzed and evaluated navigation environments in channels of Keelung and Kaohsiung, etc. through using linear

\footnotetext{
* Correspondence: xiranwang2014@163.com

${ }^{1}$ School of Navigation, Wuhan University of Technology, Wuhan, China

${ }^{2}$ Hubei Inland Shipping Technology Key Laboratory, Wuhan, China

Full list of author information is available at the end of the article
}

programming and data envelopment analysis. WANG Jie et al. [2] conducted a comprehensive study of channel navigation environment via adopting the set pair method upon analyzing channel conditions, hydrological weather, traffic, navigation supports and port supports, etc. By constructing a potential risk evaluation model, Park Y S et al. [3] carried out risk evaluations of ships navigating around South Korea's coast to achieve a scientific evaluation of navigation risk of the inbound navigation channel in $\mathrm{Bu}$ san. Pietrzykowski $\mathrm{Z}$ et al. [4] evaluated the navigation risks in restricted waters with the electronic chart system, so as to provide decision-making for ship navigation. Xiao $\mathrm{X}$ et al. [5] evaluated the navigation risks in the unknown environment based on the comprehensive safety evaluation method. Zaman et al. [6] achieved risk evaluation in the navigation channels using the comprehensive safety evaluation method with the accident-prone Malacca Strait as a research object. P.Tmcoo et al. [7] quantitatively evaluated risks of human factors with the use of Bayesian evaluation model in order to obtain objective and authentic evaluation results.

Due to the fuzziness and randomness of the safety evaluation of navigation in the channel environment, only using traditional evaluation methods may easily 
cause inaccurate results due to strong subjectivity. JIA Jinzhang et al. [8] has utilized the set-valued statistics to determine the weight of external fire evaluation indicators of coal mines, effectively reducing the impact of subjective factors on the evaluation results. Arasteh A et al. [9] studied the project portfolio based on the gray theory and the fuzzy theory to prove that the proposed methods are convenient and practical upon case study. Singh $\mathrm{R}$ et al. [10] introduced a fuzzy and gray combining algorithm to study the possible risk of failure in the distribution system; the algorithm is proved to be beneficial to the management of distribution system. Goyal S et al. [11] ranked advantages and disadvantages of advanced manufacturing systems to help managers choose the best manufacturing system with the use of the fuzzy-gray relation.

In order to reduce the uncertainty and subjectivity in determining the weights of the safety evaluation indexes of channel navigation as well as to obtain objective and accurate evaluation results, a gray fuzzy evaluation model is established in this paper through combining the set-valued statistics method with the gray fuzzy theory, which aims at providing research methods for managing the channel safety.

\section{Establish a navigation risk evaluation index system}

The channel navigation system consists of many parts with complex and changeable index factors affecting the safety of channel navigation. Therefore, it is necessary to make a detailed analysis of index factors affecting the risk of channel navigation and to find out the inherent law with an aim at studying the risk of channel navigation accurately. As there are mutual influences among each index factor, it shall study all the index factors that may affect the safety of channel navigation in a comprehensive way, rather than analyzing a single index factor unilaterally and in isolation. A total of 20 index factors of navigation risks are selected according to relevant research, see Table 1 .

Utilizing the AHP (analytical hierarchy process) [12-15], the above index factors of channel navigation are screened with SPSS, so as to select representative index factors of navigation in the inland channels. During the screening process, it must guarantee that selected index factors are representative and fully reflecting the risk information of channel navigation. After screening, an evaluation index system influencing the safety in the inland channel navigating environment is established to divide the safety factors of channel navigating environment into three types of first-level evaluation indexes including the natural environment, channel conditions, and traffic environment. Based on the first-level evaluation indexes, 12-s level evaluation indicators are divided, see Table 2.

\section{Determine weights of evaluation indexes}

Determining the weight of risk evaluation of channel navigation is an important process for evaluating the research object. At the same time, whether the weight determined is reasonable has a direct impact on the accuracy of the final evaluation results. Many scholars have studied calculation methods of index weights from both subjective and objective perspectives. After referring to related literature and comparatively analyzing weight calculation methods, two commonly used methods at present are AHP and entropy weight. Of which, AHP mainly calculates the index weight of evaluation with human's subjective experience, while the entropy weight calculates the index weight of evaluation through correlation analysis of data. It is difficult to obtain accurate index weights via using traditional weight calculation methods due to the complexity of channel navigation environment. Hence, a method of set-valued statistics is adopted in this paper to determine index weights. The method of set-valued statistics is a fuzzy interval instead of a definite value in the classical statistics, which can accurately reflect experts' scoring criteria and increase the credibility of evaluation results. Therefore, the set-valued statistics is adopted in this paper to determine the weight of each index of evaluation.

\subsection{Determine the weight with set-valued statistics}

Set value statistics $[16,17]$ is scientific and reasonable for processing the fuzzy phenomenon, which is to

Table 1 Index factors for risks of channel navigation

\begin{tabular}{llllll}
\hline No. & Index factor & No. & Index factor & No. & Index factor \\
\hline$X_{1}$ & Wind & $x_{8}$ & Width of channel & $X_{15}$ & Traffic flow \\
$X_{2}$ & Wave & $x_{9}$ & Length of channel & $X_{16}$ & Maritime control \\
$X_{3}$ & Current & $x_{10}$ & Channel bending & $X_{17}$ & Navigation facilities \\
$X_{4}$ & Tide & $X_{11}$ & Obstruction & $X_{18}$ & Management system \\
$X_{5}$ & Visibility & $X_{12}$ & Hydraulic structure & $X_{19}$ & VTS coverage \\
$X_{6}$ & Precipitation & $X_{13}$ & Turning point & $X_{20}$ & Salvage from the shore \\
$X_{7}$ & Depth of channel & $X_{14}$ & Channel intersection & & \\
\hline
\end{tabular}


Table 2 The safety index system in the inland channel navigating environment

\begin{tabular}{ll}
\hline First-level evaluation factor & Second-level evaluation factor \\
\hline Natural environment $Y_{1}$ & Wind $X_{1}$ \\
& Current $X_{3}$ \\
& Visibility $X_{5}$ \\
& Depth of channel $X_{7}$ \\
& Width of channel $X_{8}$ \\
& Channel bending $X_{10}$ \\
& Obstruction $X_{11}$ \\
& Turning point $X_{13}$ \\
& Channel intersection $X_{14}$ \\
& Traffic flow $X_{15}$ \\
& Navigation facilities $X_{17}$ \\
Traffic environment $Y_{3}$ & VTS coverage $X_{19}$ \\
\hline
\end{tabular}

quantitatively study properties of research objects. For complex objects, it is more objective and reasonable to estimate specific values by an interval. In each experiment, a specific point can be obtained by the classical statistics, while an interval can be obtained through the set-valued statistics. Assuming that the $k$ th evaluator estimates the attribute value $Z$ as $\left[Z_{1}^{k}, Z_{2}^{k}\right]$. In that case, these intervals can form a sequence, i.e., $\left[Z_{1}^{1}, Z_{2}^{1}\right],\left[Z_{1}^{2}, Z_{2}^{2}\right], \ldots,\left[Z_{1}^{l}, Z_{2}^{l}\right]$. Through covering intervals on the evaluation shaft, the following distributions can be formed:

$$
P(Z)=\frac{1}{l} \sum_{k=1}^{l} \phi\left|z_{1}^{k} \cdot z_{2}^{k}\right|(Z)
$$

Where

$$
\phi\left|z_{1}^{k} \cdot z_{2}^{k}\right|(Z)=\left\{\begin{array}{cc}
1 & \left(Z_{1}^{\mathrm{k}} \leq z \leq Z_{2}^{k}\right) \\
0 & \text { (other) }
\end{array}\right.
$$

With the $P(Z)$ function as a sample drop-shadow function, the attribute value $Z$ of the research object can be estimated as

$$
\bar{Z}=\int_{Z_{\min }}^{Z_{\max }} Z \cdot P(Z) \mathrm{d} Z / \int_{Z_{\min }}^{Z_{\max }} P(Z) \mathrm{d} Z
$$

Where, $Z_{\max }$ and $Z_{\min }$ in the equation represent the maximum value and the minimum value of $Z$, respectively. It can be seen that

$$
\int_{Z_{\min }}^{Z_{\max }} P(Z) \mathrm{d} Z=\frac{1}{l} \sum_{k=1}^{l}\left[Z_{2}^{k}-Z_{1}^{k}\right]
$$

$$
\int_{Z_{\min }}^{Z_{\max }} Z \cdot P(Z) \mathrm{d} Z=\frac{1}{2 l} \sum_{k=1}^{l}\left[\left(Z_{2}^{k}\right)^{2}-\left(Z_{1}^{k}\right)^{2}\right]
$$

Therefore,

$$
\bar{Z}=\frac{1}{2} \sum_{k=1}^{l}\left[\left(Z_{2}^{k}\right)^{2}-\left(Z_{1}^{k}\right)^{2}\right] / \sum_{k=1}^{l}\left[Z_{2}^{k}-Z_{1}^{k}\right]
$$

According to the principle of set-valued statistics, the weight of the evaluation index can be determined. In a certain evaluation index system, the evaluation index is set as $m$, its set composed is

$C=\left\{C_{1}, C_{2}, \ldots, C_{\mathrm{m}}\right\}$. Supposing there are $n$ experts involved in judging indexes, the set composed is

$P=\left\{p_{1}, p_{2}, \ldots, p_{\mathrm{n}}\right\}$. For a given evaluation index $C_{\mathrm{i}}$, the weight interval given by each expert is $\left[a_{1 \mathrm{i}}, b_{1 \mathrm{i}}\right],\left[a_{2 \mathrm{i}}, b_{2 \mathrm{i}}\right]$, $\ldots,\left[a_{\mathrm{ki}}, b_{\mathrm{ki}}\right], \ldots,\left[a_{\mathrm{ni}}, b_{\mathrm{ni}}\right]$, respectively. Regarding statistics of interval value of each evaluation index, see Table 3 .

Through calculations, the relative weight of the evaluation index $C_{\mathrm{i}}$ is

$$
\begin{aligned}
\bar{w}_{i} & =\frac{1}{2} \frac{\sum_{k=1}^{n}\left(b_{k i}^{2}-a_{k i}^{2}\right)}{\sum_{k=1}^{n}\left(b_{k i}-a_{k i}\right)}, i=1,2, \ldots, m ; k \\
& =1,2, \ldots, n
\end{aligned}
$$

If each expert's objective circumstances such as titles, academic qualifications, and lengths of service in the process of determining the weight, the weight of each expert is set as $q_{\mathrm{k}}$,

$$
\sum_{k=1}^{n} q_{k}=1
$$

Then, the relative weight of the evaluation index $C_{\mathrm{i}}$ is

Table 3 Estimated interval of the weight of the evaluation index

\begin{tabular}{lllllll}
\hline $\begin{array}{l}\text { Evaluation } \\
\text { expert }\end{array}$ & \multicolumn{6}{l}{ Evaluation index security range } \\
\cline { 2 - 7 } & $C_{1}$ & $C_{2}$ & $\ldots$ & $C_{i}$ & $\ldots$ & $C_{m}$ \\
\hline$p_{1}$ & {$\left[a_{11}, b_{11}\right]$} & {$\left[a_{12}, b_{12}\right]$} & $\ldots$ & {$\left[a_{11}, b_{1 i}\right]$} & $\ldots$ & {$\left[a_{1 m}, b_{1 m}\right]$} \\
$p_{2}$ & {$\left[a_{21}, b_{21}\right]$} & {$\left[a_{22}, b_{22}\right]$} & $\ldots$ & {$\left[a_{2 i}, b_{2 i}\right]$} & $\ldots$ & {$\left[a_{2 m}, b_{2 m}\right]$} \\
$\ldots$ & $\ldots$ & $\ldots$ & $\ldots$ & $\ldots$ & $\ldots$ & $\ldots$ \\
$p_{k}$ & {$\left[a_{k 1}, b_{k 1}\right]$} & {$\left[a_{k 2}, b_{k 2}\right]$} & $\ldots$ & {$\left[a_{k i}, b_{k i}\right]$} & $\ldots$ & {$\left[a_{k m}, b_{k m}\right]$} \\
$\ldots$ & $\ldots$ & $\ldots$ & $\ldots$ & $\ldots$ & $\ldots$ & $\ldots$ \\
$p_{n}$ & {$\left[a_{n 1}, b_{n 1}\right]$} & {$\left[a_{n 2}, b_{n 2}\right]$} & $\ldots$ & {$\left[a_{n i}, b_{n i}\right]$} & $\ldots$ & {$\left[a_{n m}, b_{n m}\right]$} \\
\hline
\end{tabular}




$$
\begin{aligned}
\bar{w}_{i} & =\frac{1}{2} \frac{\sum_{k=1}^{n} q_{k}\left(b_{k i}^{2}-a_{k i}^{2}\right)}{\sum_{k=1}^{n} q_{k}\left(b_{k i}-a_{k i}\right)}, i=1,2, \ldots, m ; k \\
& =1,2, \ldots, n
\end{aligned}
$$

After the relative weight of the index $C_{\mathrm{i}}$ calculated from Eqs. (8) and (9) is processed by normalization, the weight of the index $C_{\mathrm{i}}$ can be obtained

$$
w_{i}=\frac{\overline{w_{i}}}{\sum_{i=1}^{m} \overline{w_{i}}}
$$

Thus, the weight vectors obtained of each evaluation index in the evaluation system are

$$
W=w_{1}, w_{2}, \ldots, w_{i}, \ldots, w_{m}
$$

\subsection{Reliability analysis of index weight}

The reliability analysis should be also conducted upon weights opened by the set-valued statistics. The method of interval variance is used in this paper to analyze the reliability of weights. For the index $\mathrm{Ci}$, the variance of its weight interval is defined as

$$
\begin{aligned}
F_{i} & =\frac{\sum_{k=1}^{n}\left[\left(b_{k i}-\bar{w}_{i}\right)^{3}-\left(a_{k i}-\bar{w}_{i}\right)^{3}\right]}{3 \sum_{k=1}^{n}\left(b_{k i}-a_{k i}\right)}, i \\
& =1,2, \ldots, m ; k=1,2, \ldots, n
\end{aligned}
$$

The larger the Fi, the lower the reliability of the weight of the evaluation index is indicated; the smaller the $\mathrm{Fi}$, the higher the reliability of the weight of the evaluation index is indicated.

\section{Methods section}

The gray-fuzzy evaluation model is an evaluation model based on the gray system theory and the fuzzy mathematics theory. At present, the model is widely used in risk evaluation, effectively resolving a series of ambiguities and uncertainties. In view of the fuzziness and uncertainty of the channel navigation system, a gray-fuzzy risk evaluation model of channel navigation is built in this chapter on the basis of the gray system theory and the fuzzy mathematics theory, so that theoretical supports can be provided for evaluating the risk of navigation channel and ensuring the navigation safety of that channel.

\subsection{The gray system theory}

The gray system theory was first proposed by Chinese mathematician Prof. DENG Julong [18] based on the concept of "gray box," which has gradually developed into a new discipline as it is put forward. As the gray system theory has not only been theoretically innovated, but also made great breakthroughs in thinking compared with the traditional mathematical research methods, it has been widely applied in the economy, society, agriculture, transportation, and various field with. Moreover, significant applied results have been achieved by solving numerous problems. In the gray system theory, the gray number is a fundamental concept. It is usually used to represent a fuzzy range, which is not a precise numerical value and can be expressed with " $\otimes$." A whitening process should be conducted on the gray number in order to quantitatively describe the gray number in a determined and clear way. For the gray number $[a, b]$ at any interval, its whitening value is expressed as

$$
\tilde{\otimes}=\alpha a+(1-\alpha) b, \alpha \in[0,1]
$$

As a core of the gray system theory, the whitening weight function is an important step in the gray research. At present, there are three common types of whitening weight functions, i.e., upper-level, intermediate, and lower-level; specific shape functions and expressions are presented as follows:

(1) The upper-level whitening weight function, the gray number $\otimes$ is $\left[l_{1},+\infty\right]$; and Fig. 1 of the function and the equation are presented as follows:

$$
f_{1}\left(l_{s j}\right)= \begin{cases}l_{s j} / l_{1} & l_{s j} \in\left[l_{1},+\infty\right) \\ 1 & l_{s j} \in\left[l_{1},+\infty\right) \\ 0 & l_{s j} \in[-\infty, 0)\end{cases}
$$

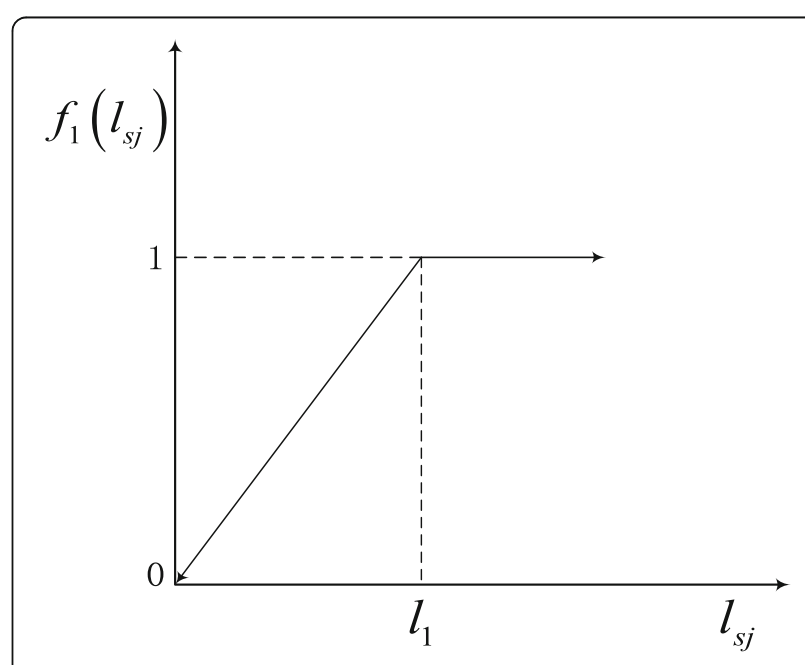

Fig. 1 Upper-level whitening weight function 


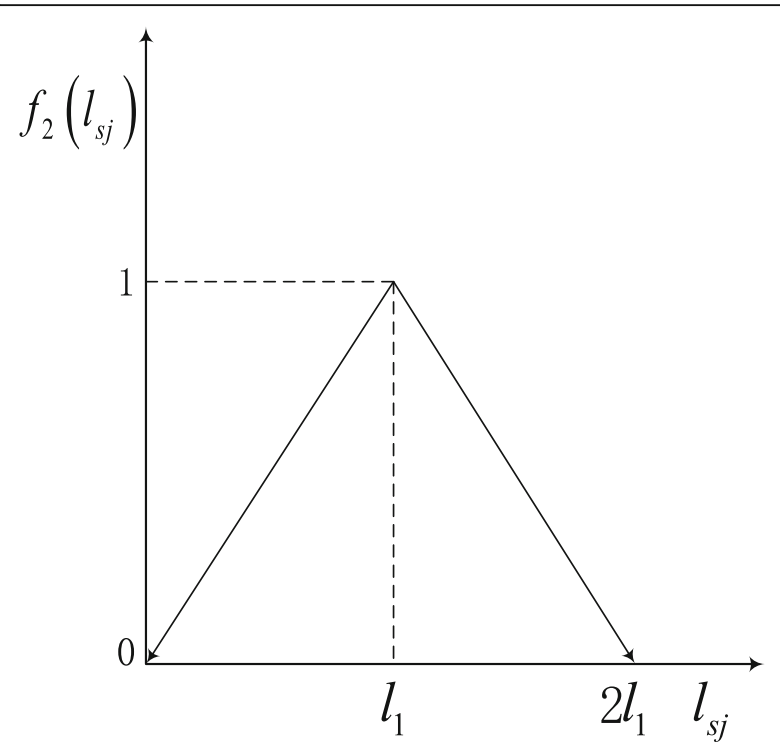

Fig. 2 Intermediate whitening weight function

(2) The intermediate whitening weight function, the gray number $\otimes$ is $\left[0, l_{1}, 2 l_{2}\right]$; and Fig. 2 of the whitening weight function and the equation are presented as follows:

$$
f_{2}\left(l_{s j}\right)=\left\{\begin{array}{lc}
l_{s j} / l_{1} & l_{s j} \in\left[0, l_{1}\right) \\
2-l_{s j} / l_{1} & l_{s j} \in\left[l_{1},+\infty\right) \\
0 & l_{s j} \notin\left[0,2 l_{1}\right)
\end{array}\right.
$$

(3) The lower-level whitening weight function, the gray number $\otimes$ is $\left[0, l_{1}, l_{2}\right]$; and Fig. 3 of the whitening weight function and the equation are presented as follows:

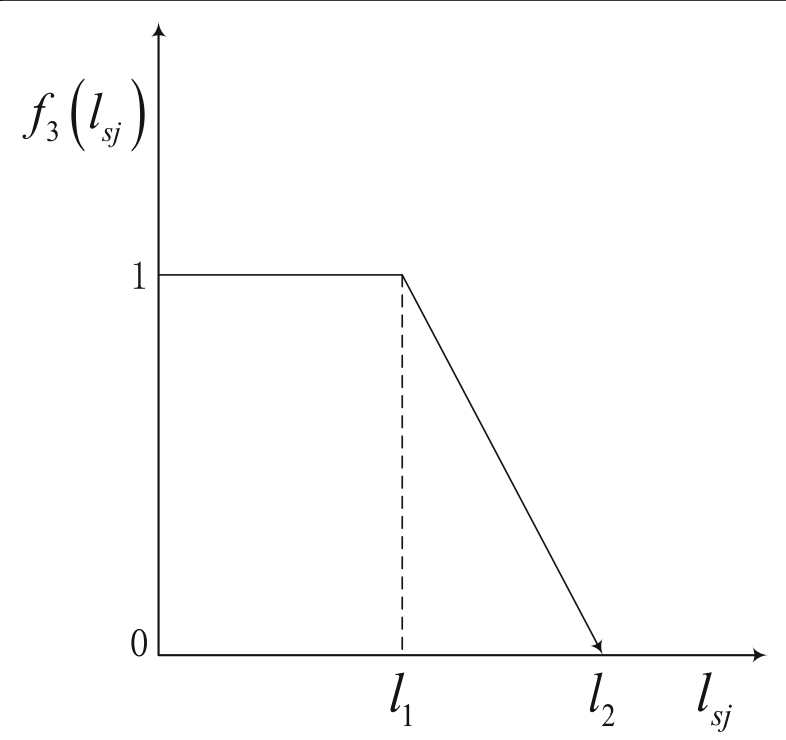

Fig. 3 Intermediate whitening weight function

$$
f_{3}\left(l_{s j}\right)=\left\{\begin{array}{lc}
1 & l_{s j} \in\left[0, l_{1}\right) \\
l_{2}-l_{s j} / l_{2}-l_{1} & l_{s j} \in\left[l_{1}, l_{2}\right) \\
0 & l_{s j} \notin\left[0, l_{2}\right)
\end{array}\right.
$$

\subsection{Construct a gray-fuzzy evaluation model}

The navigation channel risk evaluation system is a complex system with many influencing factors for risk research of the navigation channel $[19,20]$. At the same time, there are also mutual influences among factors; so, there is a great ambiguity in the risk evaluation of navigation. Furthermore, as relevant data are insufficient enough, it is difficult to analyze the risk of navigation channel with classical mathematical statistics Therefore, both advantages are integrated in this paper to build a gray-fuzzy evaluation model; the model structure is shown in Fig. 4.

A channel navigation risk evaluation model is established according to the above gray-fuzzy evaluation model. Steps of model building are presented as follows:

\subsection{1 (1) Construct a sample matrix}

According to the determined navigation risk evaluation index system [21, 22], a risk evaluation model channel navigation is constructed. The gray-fuzzy evaluation method is used to evaluate the safety evaluation for the evaluation index $C$. The set of first-level evaluation indexes is set as $C=\left\{C_{1}, C_{2}, \ldots, C_{\mathrm{i}}, \ldots, C_{\mathrm{s}}\right\}$; where, $i=1,2$, ..., $s$. The set of second-level evaluation indexes is $C_{\mathrm{i}}$ $=\left\{C_{\mathrm{i} 1}, C_{\mathrm{i} 2}, \ldots, C_{\mathrm{ij}}, \ldots, C_{\mathrm{it}}\right\}$ ( $t$ is the number belonging to the first-level evaluation indexes in the second-level evaluation indexes); where, $j=1,2, \ldots, t$.

$n$ experts score each evaluation index in the index set $C_{\mathrm{i}}=\left\{C_{\mathrm{i} 1}, C_{\mathrm{i} 2}, \ldots, C_{\mathrm{ij}}, \ldots, C_{\mathrm{it}}\right\}$ of second-level indexes according to the criteria, i.e., $d_{\mathrm{ijk}}(k=1,2, \ldots, n) . \mathrm{d}_{\mathrm{ijk}}$ is utilized to build an evaluation sample matrix:

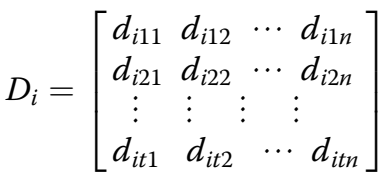

\subsection{2 (2) Establish a whitening weight function}

Upon comprehensively studying the characteristics of navigation channel, the gray type of environmental risk evaluation of navigation channel can be divided into five levels [23, 24], of which, levels 1, 2, 3, 4, and 5 of each evaluation gray class are corresponding to dangerous, slightly dangerous, general, relatively safe, and safe, respectively. At the same time, the corresponding threshold of each gray type can be determined according to the gray class, as shown in Table 4. 


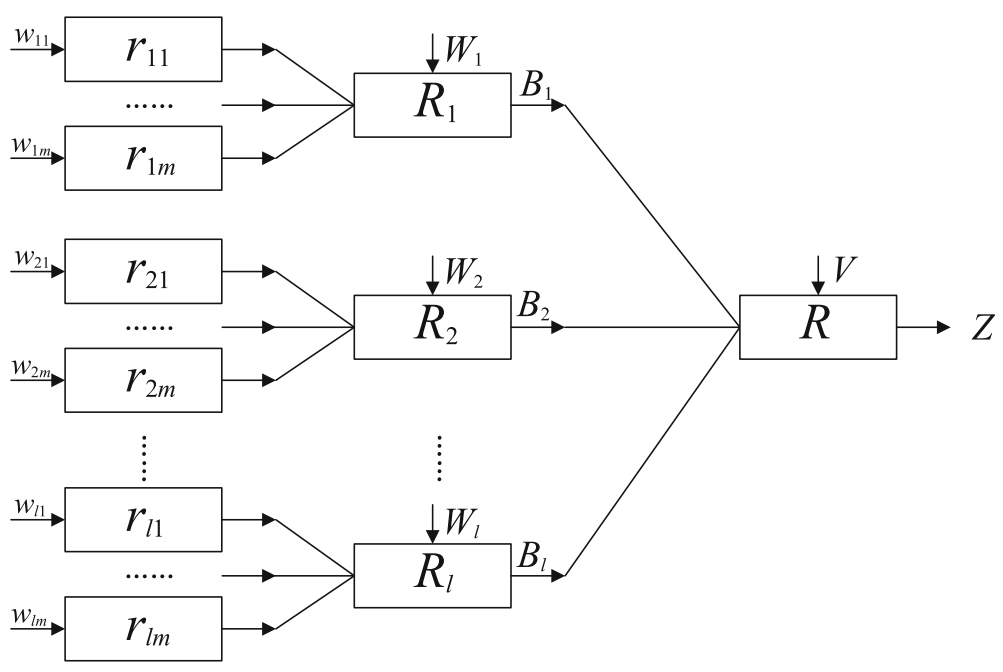

Fig. 4 The gray-fuzzy evaluation model

Based on the evaluation gray class and the corresponding threshold, the whitening weight function is established as

$$
\begin{aligned}
& f_{1}= \begin{cases}\frac{1}{10-d_{i j k}} & d_{i j k} \in[0,1) \\
9 & d_{i j k} \in[1,10]\end{cases} \\
& f_{2}= \begin{cases}\frac{1}{3} d_{i j k} & d_{i j k} \in[0,3) \\
\frac{10-d_{i j k}}{7} & d_{i j k} \in[3,10]\end{cases} \\
& f_{3}= \begin{cases}\frac{1}{5} d_{i j k} & d_{i j k} \in[0,5) \\
\frac{10-d_{i j k}}{5} & d_{i j k} \in[5,10]\end{cases} \\
& f_{4}= \begin{cases}\frac{1}{7} d_{i j k} & d_{i j k} \in[0,7) \\
\frac{10-d_{i j k}}{3} & d_{i j k} \in[7,10]\end{cases} \\
& f_{5}= \begin{cases}\frac{d_{i j k}}{9} & d_{i j k} \in[0,9) \\
1 & d_{i j k} \in[9,10]\end{cases}
\end{aligned}
$$

\subsection{3 (3) Construct a second-level gray-fuzzy evaluation} matrix

According to each expert's scored results $d_{\mathrm{ijk}}$ on the second-level evaluation index $C_{\mathrm{ij}}$, its evaluation coefficient in the eth evaluation gray is calculated as

Table 4 Threshold corresponding to each gray class

\begin{tabular}{cccccc}
\hline Gray class & $e=1$ & $e=2$ & $e=3$ & $e=4$ & $e=5$ \\
Threshold & 1 & 3 & 5 & 7 & 9 \\
\hline
\end{tabular}

$$
y_{i j e}=\sum_{k=1}^{n} f_{e}\left(d_{i j k}\right)
$$

The sum of the evaluation coefficients of second-level evaluation index $C_{\mathrm{ij}}$ in each evaluation gray class can be described as

$$
Y_{i j}=\sum_{e=1}^{g} y_{i j e}
$$

The evaluation coefficient of each gray class is calculated before conducting a normalized processing. The gray evaluation weight obtained can be denoted as

$$
r_{i j e}=\frac{y_{i j e}}{Y_{i j}}
$$

Each gray evaluation weight in the evaluation index $C_{\mathrm{i}}$ are counted to establish a second-level gray-fuzzy evaluation matrix $r_{\mathrm{i}}$ :

$$
r_{i}=\left[\begin{array}{cccc}
\mathrm{r}_{i 11} & r_{i 12} & \cdots & r_{i 1 g} \\
r_{i 21} & r_{i 22} & \cdots & r_{i 2 g} \\
\vdots & \vdots & \vdots & \vdots \\
r_{i t 1} & r_{i t 2} & \cdots & r_{i t g}
\end{array}\right]
$$

\subsection{4 (4) Calculate first-level index evaluation results}

When calculating evaluation results of the first-level evaluation indexes, each weight $W_{\mathrm{i}}=\left(w_{\mathrm{i} 1}, w_{\mathrm{i} 2}, \ldots, w_{\mathrm{ij}}, \ldots\right.$, $w_{\text {it }}$ ) of the second-level evaluation indexes multiplied by the corresponding second-level gray-fuzzy evaluation matrix $r_{\mathrm{i}}$, respectively, can obtain the corresponding evaluation results os first-level indexes, i.e., $R_{\mathrm{i}}=W_{\text {iri }}$. 


\subsection{5 (5) Calculate the comprehensive evaluation result}

The calculation method of the evaluation result of the first- level index $C_{\mathrm{i}}$ is utilized to obtain the final evaluation result $R$ of the total $\operatorname{target} C$, i.e., $R=$ Wr. According to the principle of maximum membership degree, the maximum value of the evaluation result $R$ is obtained in $C$, whose corresponding gray class is the comprehensive evaluation result.

\section{Experimental section}

An inland channel in Guangdong belongs to a part of the main channel for marine outfall of the Guangzhou port, which is located in the east of Haixinsha of Shiziyang with Dahu Channel to the south, east, and west channels of Lianhua Mountain to the north. The channel is laid in U-shape with a total length of $8.6 \mathrm{~km}$. Relevant parameters see Table 5.

\subsection{Determination of evaluation index weights}

According to the determined index system of environmental safety evaluation of channel navigation in Table 2, evaluation indexes at all levels are set as $Y=\left\{Y_{1}, Y_{2}, Y_{3}\right\}$, $Y_{1}=\left\{X_{1}, X_{3}, X_{5}\right\}, Y_{2}=\left\{X_{7}, X_{8}, X_{10}, X_{11}, X_{13}, X_{14}\right\}, Y_{3}=\left\{X_{15}\right.$, $\left.X_{17}, X_{19}\right\}$. The weight interval is set as $[0,10]$, in order to facilitate the experts to accurately determine the weight of each evaluation index. Analytic hierarchy process (AHP) is employed to set weights of five experts (two captains and three senior engineers) of the university, shipping company, maritime bureau, channel department, and design institute. The weights of all kinds of experts are respectively $Q=(0.20,0.15,0.25,0.30,0.10)$. The estimated value of each evaluation index in Table 6. In this paper, natural environment $Y_{1}$, channel conditions $Y_{2}$ and traffic environment $Y_{3}$ in the first-level evaluation index are taken as examples to calculate the weight.

According to the Eq. (9), the relative weight $\bar{w}=(3.61$, $7.02,2.16)$ of first-level indexes including natural environment, channel condition, and traffic environment are calculated. With the Eq. (10), the relative weight solved is processed in normalization to determine the final

Table 5 Parameters of navigation in the Guangdong inland channel

\begin{tabular}{ll}
\hline Index factor & Channel parameters \\
\hline Wind & $0.3948 \mathrm{~m} / \mathrm{s}$ \\
Current & $0.0943 \mathrm{~m} / \mathrm{s}$ \\
Width of channel & $160 \mathrm{~m}$ \\
Length of channel & $8596 \mathrm{~m}$ \\
Turning point $V$ & $\mathrm{~V}-29^{\circ}$ \\
Turning point $U$ & $\mathrm{U}-21^{\circ}$ \\
Coverage of the navigation aid facility & $90 \%$ \\
\hline
\end{tabular}

Table 6 Evaluation index security range

\begin{tabular}{lllcc}
\hline $\begin{array}{llll}\text { Evaluation } \\
\text { expert }\end{array}$ & $\begin{array}{l}\text { Evaluation } \\
\text { expert } \\
\text { weight }\end{array}$ & \multicolumn{3}{l}{ Evaluation index security range } \\
\cline { 4 - 5 } & 0.2 & {$[2.9,4.1]$} & {$[7.4,7.7]$} & {$[2.8,3.4]$} \\
\hline$P_{1}$ & 0.15 & {$[3.1,3.6]$} & {$[6.7,7.4]$} & {$[2.1,2.5]$} \\
$P_{2}$ & 0.25 & {$[3.6,4.1]$} & {$[6.2,7.1]$} & {$[2.4,2.7]$} \\
$P_{3}$ & 0.30 & {$[4.2,4.5]$} & {$[7.0,7.1]$} & {$[2.6,3.1]$} \\
$P_{4}$ & 0.10 & {$[4.4,4.8]$} & {$[6.9,7.1]$} & {$[3.0,3.5]$} \\
$P_{5}$ & &
\end{tabular}

weight of the three first-level indexes $W=(0.274,0.501$, 0.212 ).

Likewise, weights of all kinds of second-level indexes in the index system can be also solved. Calculated results of weight calculation for all kinds of second-level indexes are $W_{1}=(0.231,0.452,0.281), W_{2}=(0.156,0.212$, $0.131,0.120,0.171,0.157)$, and $W_{3}=(0.271,0.414$, 0.312 ), respectively.

It is necessary to test the index weights solved in order to determine whether the weight is reasonable. Also, the reliability of index weight can be tested according to the interval variance method; where, $F=(0.253,0.121$, $0.124)$. The test results show that the reliability of the index weight of the traffic environment is relatively high, while the natural environment index is relatively low.

\subsection{Determine the evaluation sample matrix}

On the basis of calculating the weight of the index, the evaluation model is used for calculating and analyzing the risk of channel navigation [25]. Experts are invited to score second-level indexes and to establish a scoring standard with a scoring range of $[0,10]$. Each expert score evaluation indexes and count scores based on the actual situation of the inland channel. Based on the above research results, analyzing steps for conducting navigation risk evaluation in the research are presented as follows:

\subsection{1 (1) Determine the evaluation coefficient in the evaluation of gray class}

For the second-level index $X_{1}$, when $e=1$ according to the Eq. (24),

$$
y_{111}=f_{1}\left(d_{111}\right)+f_{1}\left(d_{112}\right)+\ldots+f_{1}\left(d_{11 n}\right)=37.136 \text {. }
$$

Similarly, when $e=2$, y112 $=47.766$; when $e=3$, y113 $=66.880 ;$ when $e=4, y 114=77.950 ;$ and when $e=5$, y115 $=60.614$. On the basis of Eqs. ((25) and (26), the evaluation coefficient corresponding to each evaluation gray class is further calculated and normalized to calculate the weight vector of gray evaluation,

$r_{11}=(0.117,0.161,0.232,0.168,0.210)$

and $X_{5}$ are calculated respectively as

$r_{12}=(0.071,0.138,0.213,0.351,0.222)$

$r_{13}=(0.091,0.114,0.136,0.233,0.416)$. 


\subsection{2 (2) Determine the gray-fuzzy evaluation matrix}

According to the obtained gray evaluation vector, the gray-fuzzy evaluation matrix is established as

$$
r_{1}=\left[\begin{array}{lllll}
0.117 & 0.161 & 0.232 & 0.168 & 0.210 \\
0.071 & 0.138 & 0.213 & 0.351 & 0.222 \\
0.091 & 0.114 & 0.136 & 0.233 & 0.416
\end{array}\right]
$$

Similarly, according to the above method, the gray-fuzzy evaluation matrix of other first-level evaluation indexes are

$$
\begin{aligned}
& r_{2}=\left[\begin{array}{lllll}
0.117 & 0.161 & 0.232 & 0.168 & 0.210 \\
0.141 & 0.183 & 0.255 & 0.233 & 0.181 \\
0.147 & 0.186 & 0.253 & 0.221 & 0.172 \\
0.149 & 0.150 & 0.210 & 0.290 & 0.222 \\
0.132 & 0.124 & 0.211 & 0.315 & 0.256 \\
0.166 & 0.216 & 0.256 & 0.215 & 0.151
\end{array}\right] \\
& r_{3}=\left[\begin{array}{lllll}
0.152 & 0.195 & 0.274 & 0.186 & 0.171 \\
0.113 & 0.132 & 0.187 & 0.287 & 0.254 \\
0.092 & 0.126 & 0.167 & 0.272 & 0.353
\end{array}\right]
\end{aligned}
$$

\subsection{3 (3) Determine evaluation results of first-level indicating evaluation}

According to the method of calculating first-level index evaluation results, the first-level evaluation index evaluation results $R_{\mathrm{i}}=W_{\text {iri }}$ are obtained as

$$
\begin{aligned}
& R_{1}=(0.091,0.132,0.193,0.287,0.281) \\
& R_{2}=(0.125,0.173,0.240,0.252,0.187) \\
& R_{3}=(0.115,0.142,0.218,0.243,0.256) .
\end{aligned}
$$

\subsection{Calculation and analysis of evaluation results of the comprehensive system model}

According to the first-level evaluation result $R_{\mathrm{i}}$, a comprehensive gray-fuzzy evaluation matrix of navigation risk in the inland channel in Guangdong is established.

$$
r=\left[\begin{array}{lllll}
0.088 & 0.142 & 0.185 & 0.287 & 0.281 \\
0.123 & 0.181 & 0.234 & 0.253 & 0.187 \\
0.121 & 0.144 & 0.218 & 0.259 & 0.266
\end{array}\right]
$$

According to the evaluation results of first-level indexes, a comprehensive gray-fuzzy evaluation matrix is established. According to the method of calculating results of first-level index evaluation, the final evaluation result of the channel can be obtained as

$R=W r=(0.113,0.154,0.210,0.270,0.232)$.

The maximal value in the comprehensive evaluation result of channel safety in the inland channel in Guangdong is $\beta_{4}=0.252$. In that case, the gray class of the evaluation results obtained is 4 according to the principle of maximum membership degree in the fuzzy theory, which indicates that the navigation environment of the channel is "relatively safe."

\section{Conclusions}

The set-valued statistics is combined with the gray-fuzzy theory in this paper. The set-valued statistics is utilized to determine the index weight of the navigation system of the inland channel, reducing the influence of subjective factors on the evaluation results of the inland channel; also, the gray-fuzzy theory is utilized to quantitatively process the evaluation indexes, achieving the evaluation indexes transforming from qualitatively to quantitatively. Finally, an environmental safety evaluation model of navigation channel based on the set-valued statistics and the gray-fuzzy theory has been established. Moreover, the evaluation system model was adopted to calculate and analyze the environmental safety of navigation in the inland channel in Guangdong. The evaluation result obtained was relatively safe, being consistent with the actual situation. Besides, the method is highly operable in practical applications due to its objective and accurate evaluation results in comparison to traditional evaluation methods.

\section{Acknowledgements}

The research presented in this paper was supported by the Natural Science Foundation of China, and Research and Innovation Team of WTCC.

\section{Funding}

The authors acknowledge the Natural Science Foundation of China "Research on Mechanism of Ship-Entropy Catastrophe Response to Seafarer-Ship-Environment Disadjust" (51379170), Research and Innovation Team of WTCC(CX2018A04).

\section{Availability of data and materials \\ The simulation code can be downloaded at Guangdong Maritime Safety Administration.}

\section{Authors' contributions}

WYF is the main writer of this paper. He proposed the main idea, deduced the a risk evaluation model of the channel navigation, completed the simulation, and analyzed the result. HLW introduced the set-valued statistics and the gray theory. JMM collected data about the channel in Guangdong Province. All authors read and approved the final manuscript.

Competing interests

The authors declare that they have no competing interests.

\section{Publisher's Note}

Springer Nature remains neutral with regard to jurisdictional claims in published maps and institutional affiliations.

\section{Author details}

${ }^{1}$ School of Navigation, Wuhan University of Technology, Wuhan, China. ${ }^{2}$ Hubei Inland Shipping Technology Key Laboratory, Wuhan, China. ${ }^{3}$ Wuhan Technical College of Communications, Wuhan, China. ${ }^{4}$ Taizhou Maritime Bureau, Taizhou, China.

Received: 12 February 2018 Accepted: 30 May 2018 Published online: 15 June 2018

\section{References}

1. HC Wang, HS Lee, Evaluating navigation safety for harbors in Taiwan: an empirical study. J. Mar. Eng. Technol. 11(3), 31-37 (2012) 
2. J Wang, F Li, Port channel navigation safety assessment of based on RS-SPA. J. Dalian Marit. Univ. 38(1), 37-40 (2012)

3. YS Park, JS Kim, V Aydogdu, A study on the development the maritime safety assessment model in Korea waterway. J Korean Navigation Port Res. 37(6), 567-574 (2013)

4. Z Pietrzykowski, M Wielgosz, Navigation safety assessment in the restricted area with the use of ECDIS. Transnav Int. J Mar Navigation Saf Sea Transportation 5(1), 29-35 (2011)

5. X Xiao, M Huang, Z Cai, FSA-based navigation strategy for mobile robots under unknown environments. Comput Meas Control 15(11), 1618-1620 (2007)

6. MB Zaman, A Santoso, E Kobayashi, et al., Formal safety assessment (FSA) for analysis of ship collision using AlS data. Transnav Int J Mar Navigation Saf Sea Transportation 9(1), 67-72 (2015)

7. P Trucoo, E Cagno, E Ruggeri, A Bayesian belief network modeling of organizational factors in risk analysis: a case study in maritime transportation. Reliab. Eng. Syst. Saf. 93(6), 845-856 (2008)

8. J Jinzhang, D Xiaolei, Fuzzy evaluation and statistically oriented method in analyzing the external causes of coal mine fire. J. Saf. Environ. 15(2), 11-14 (2015)

9. A Arasteh, A Aliahmadi, MM Omran, Application of Gray Systems and fuzzy sets in combination with real options theory in project portfolio management. Arab. J. Sci. Eng. 39(8), 6489-6506 (2014)

10. Singh R, Mehfuz S, Kumar P. Intelligent decision support algorithm for distribution system restoration. Springer Plus 5(1):1175-1191(2016).

11. S Goyal, S Grover, Applying fuzzy grey relational analysis for ranking the advanced manufacturing systems. Grey Systems 2(2), 284-298 (2012)

12. KY Oang, C Yang, S Muniyappan, et al., SVD-aided pseudo principalcomponent analysis: A new method to speed up and improve determination of the optimum kinetic model from time-resolved data. Struct Dyn 4(4), 044013 (2017)

13. MA Kramer, Nonlinear principal component analysis using auto associative neural networks. AlCHE J. 37(2), 233-243 (2010)

14. H Zou, T Hastie, R Tibshirani, Sparse principal component analysis[]]. J Comput Graphical Stat 15(2), 265-286 (2006)

15. VL Skrobot, EVR Castro, RCC Pereira, et al., Use of principal component analysis (PCA) and linear discriminant analysis (LDA) in gas chromatographic (GC) data in the investigation of gasoline adulteration. Energy Fuel 21(6) 5-19 (2016)

16. Rojas C A M, Alvan R M, Carrasco-Olivera D, Topological entropy for setvalued maps. Discrete and Continuous Dynamical Systems-Series B 20(10), 3461-3474 (2017)

17. Yu G L, Topological properties of Henig globally efficient solutions of setvalued problems. Numerical Algebra Control \& Optimization 4(4), 309-316 (2017)

18. Zheng G, Grey model for prediction of container shipment. Navigation of China 37(2), 118-121 (2014)

19. L Jun, XU Zhi-jin, TANG Bei-bei, Prediction method of ship flow based on grey-neural network improved by GA. Ship \& Ocean Engineering 42(5), 135-137 (2013)

20. J Ming-ming, X Xi-long, H Li-wen, L Lu, Safety evaluation model for the water-way navigation based on the centralized statistical method-grey fuzzy. J Saf Environ 17(1), 41-45 (2017)

21. T Chai, J Weng, X De-qi, Development of a quantitative risk assessment model for ship collisions in fairways. Saf. Sci. 91, 71-83 (2017)

22. A Bela, H Le Sourne, L Buldgen, P Rigo, Ship collision analysis on offshore wind turbine monopile foundations. Mar. Struct. 51, 220-241 (2017)

23. A Graziano, AP Teixeira, C Guedes Soares, Classification of human errors in grounding and collision accidents using the TRACEr taxonomy. Safety Science 86, 245-257 (2016)

24. R Szlapczynski, J Szlapczynska, An analysis of domain-based ship collision risk parameters. Ocean Eng. 126, 47-56 (2016)

25. P Sotiralis, NP Ventikos, R Hamann, P Golyshev, AP Teixeira, Incorporation of human factors into ship collision risk models focusing on human centred design aspects. Reliab. Eng. Syst. Saf. 156, 210-227 (2016)

\section{Submit your manuscript to a SpringerOpen ${ }^{\circ}$ journal and benefit from:}

- Convenient online submission

- Rigorous peer review

- Open access: articles freely available online

- High visibility within the field

- Retaining the copyright to your article

Submit your next manuscript at $\gg$ springeropen.com 